\title{
Optimization of the Nutrient Medium for Flammulina velutipes Submerged Biomass Production and Micromorphology of Its Mycelium
}

\author{
Larissa Krasnopolskaya *(D), Maria Shuktueva, Aleksandr Golyshkin, Nailya Almyasheva (D) and Maria Yarina
}

check for

updates

Citation: Krasnopolskaya, L.; Shuktueva, M.; Golyshkin, A.; Almyasheva, N.; Yarina, M. Optimization of the Nutrient Medium for Flammulina velutipes Submerged Biomass Production and Micromorphology of Its Mycelium. Fermentation 2021, 7, 180. https:// doi.org/10.3390/fermentation7030180

Academic Editors: Kurt A. Rosentrater and Diomi Mamma

Received: 5 July 2021

Accepted: 1 September 2021

Published: 5 September 2021

Publisher's Note: MDPI stays neutral with regard to jurisdictional claims in published maps and institutional affiliations.

Copyright: (c) 2021 by the authors. Licensee MDPI, Basel, Switzerland. This article is an open access article distributed under the terms and conditions of the Creative Commons Attribution (CC BY) license (https:// creativecommons.org/licenses/by/ $4.0 /)$.
Institute of New Antibiotics, 119021 Moscow, Russia; mleonteva@yandex.ru (M.S.); pilym365@mail.ru (A.G.); almyashevanelya@mail.ru (N.A.); maria.s.yarina@gmail.com (M.Y.)

* Correspondence: lmkrasnopolska@gmail.com

\begin{abstract}
Based on the assessment of the trophic needs of basidiomycete Flammulina velutipes and the optimization of the composition of the nutrient medium using design of experimental approach, the yield of the submerged biomass of the fungus was increased to $41 \mathrm{~g} / \mathrm{L}$, the duration of the cultivation process was reduced to 5 days. For this purpose, the $2^{4}$ full factorial design, the BoxWilson steep ascent method and the construction of the response surface were used. Using the method of scanning electron microscopy, it was shown that the studied F. velutipes strain grew in the form of small spherical pellets with a diameter of 1-2 $\mathrm{mm}$ on an optimized medium. The surface of the pellets was loose; the inner part was filled with hyphae tightly adjacent to each other. The center of the pellets had no cavity. F. velutipes pellets were formed by septate hyphae with clamp connections. The micromorphological characteristics of the submerged $F$. velutipes mycelium ensured a high diffusion of nutrients and oxygen into the pellets and their maximum filling of the volume of the culture medium.
\end{abstract}

Keywords: Flammulina velutipes; submerged cultivation; design of experiment; yield of biomass; micromorphology of the mycelium

\section{Introduction}

Active research on culinary-medicinal basidiomycetes is associated with their ability to synthesize metabolites of various chemical nature and biological effects. One of the most popular species of this group of macromycetes is Flammulina velutipes (Curtis) Singer or winter mushroom. Due to its high nutritional value and flavorful taste, the fruit bodies of F. velutipes are grown on a large scale using solid-state cultivation. Fruiting bodies and vegetative mycelium of $F$. velutipes contain biologically active metabolites: polysaccharides, peptides, and sesquiterpenes. Polysaccharides of F. velutipes possess antioxidant, immunomodulatory, anti-tumor, anti-inflammatory, antiviral, cognitive-enhancing, hepatoprotective and some other properties [1-4]. Peptides exhibiting immunomodulatory properties were isolated from F. velutipes [5]. Biologically active metabolites of F. velutipes with low molecular weight are mainly represented by sesquiterpenes, which display antibacterial, antifungal, antimalarial, or cytotoxic activity [6].

In our experiments, the F. velutipes strain was selected as a promising producer of biologically active polysaccharides. In experiments, the total fraction of water-soluble polysaccharides from the submerged mycelium of this strain inhibited the development of a transplanted solid tumor of murine leukemia P388. The maximum inhibition of tumor growth by the water-soluble polysaccharides reached $94 \%$, with the dose of watersoluble polysaccharides at $2 \mathrm{mg} / \mathrm{kg}$ /day. Additionally, the total fraction of water-soluble polysaccharides from $F$. velutipes mycelium and its ethanol extract demonstrated antioxidant activity $[7,8]$. 
Submerged cultivation of basidiomycetes is an effective way to obtain raw materials for the isolation of their biologically active metabolites. Submerged cultivation has several advantages. Cultivation processes are carried out under sterile, strictly controlled and accurately reproducible conditions. During the submerged cultivation of the producer, it is possible to introduce additional nutrition sources, regulate $\mathrm{pH}$, temperature, aeration and agitation. The duration of submerged cultivation of basidiomycetes is several times shorter than the duration of solid-phase cultivation. To increase the efficiency of submerged cultivation of mushrooms, it is rational to optimize the cultivation conditions, primarily the composition of the nutrient medium, using the methods of mathematical design of the experiment. Filamentous basidiomycetes grow in submerged culture as dispersed mycelium or spherical mycelial units called pellets. By establishing the dependence of the submerged mycelium's morphological characteristics (type of growth, size and shape of pellets, features of their outer and inner layers, etc.) on the cultivation conditions, we create an additional tool for optimizing submerged cultivation of producers.

The aim of this work was to increase the biomass yield of the selected F. velutipes strain by optimizing the qualitative and quantitative composition of the nutrient medium and to study the micromorphology of the mycelium grown on such an optimized nutrient medium.

\section{Material and Methods}

\subsection{Chemicals}

D-glucose, agar, peptone, yeast extract, potassium dihydrogen phosphate and magnesium sulfate were purchased from Dia-M (Moscow, Russia). Glutaraldehyde was purchased from Sigma-Aldrich (St. Louis, MO, USA). Soybean oil (Moscow, Russia), soybean meal (Moscow, Russia) and corn extract (Krasnodar, Russia) were of food grade.

\subsection{Fungal Strain and Cultivation Condition}

F. velutipes strain 1 and Grifola frondosa strain 5 from the INA culture collection of Gauze Institute of New Antibiotics were used in this study. Stock cultures were maintained at $4{ }^{\circ} \mathrm{C}$ on potato-glucose agar.

The seed cultures were grown at $25{ }^{\circ} \mathrm{C}$ for 3 days on a rotary shaker at $220 \mathrm{rpm}$ in $750 \mathrm{~mL}$ Erlenmeyer flasks filled with $100 \mathrm{~mL}$ of nutrient medium containing $(\mathrm{g} / \mathrm{L}$ of tap water): 20.0 glucose; 10.0 soybean meal; 2.5 potassium hydrophosphate and 0.25 magnesium sulfate. The seed liquid medium was inoculated by mycelial agar plugs ( $3 \mathrm{~mm}$ diam.) containing seven-day-old agar cultures of basidiomycetes.

Submerged cultivation of mushroom cultures was carried out on a rotary shaker at $220 \mathrm{rpm}$ and $25^{\circ} \mathrm{C}$ in $750 \mathrm{~mL}$ Erlenmeyer flasks containing $100 \mathrm{~mL}$ of nutrient medium inoculated with $10 \mathrm{~mL}$ of liquid seed culture. The nutrient medium for submerged cultivation of $G$. frondosa contained (g/L of tap water): 20.0 soybean oil; 10.0 soybean meal; 2.5 potassium hydrophosphate and 0.25 magnesium sulfate. Following cultivation, the mycelium was separated from the culture liquid by filtration, washed twice with distilled water, and dried to a constant weight at a temperature not exceeding $45^{\circ} \mathrm{C}$. The residual moisture content in the mycelium was 5.8-6.1\%. The fungus biomass grown in soybean oil was preliminarily washed with ethanol to remove the oil. The utilization of soybean meal was monitored using light microscopy, and the biomass and culture filtrate were studied. The Olympus BX41 light microscope was used for this purpose.

\subsection{Experimental Design and Statistical Analysis}

The composition of the nutrient medium for submerged cultivation of F. velutipes was optimized using $2^{4}$ full factorial designs and the Box-Wilson steep ascent method [9]. The dry submerged biomass yield was taken as the dependent variable or response. The effect of every factor was determined by Student's test, the statistical significance of a model was evaluated by Fisher's test. Data analyses were performed using Statistica 10.0 (StatSoft Inc., 2007, Tulsa, OK, USA) 


\subsection{Scanning Electron Microscopy (SEM)}

The morphological characteristics of the submerged mycelium of $F$. velutipes were observed by SEM (TM3000, Hitachi, Tokyo, Japan). Submerged mycelium was fixed with glutaraldehyde ( $2 \%$ dissolved in $0.1 \mathrm{M}$ phosphate buffer (PBS)) for $2 \mathrm{~h}$ at $4{ }^{\circ} \mathrm{C}$. Samples were washed with PBS and dehydrated with 30, 50, 70 and $96 \mathrm{wt}$ \% ethanol at $15 \mathrm{~min}$ intervals and dried following critical point drying procedure. Dried samples were covered by a thin gold layer.

\subsection{Total Lipid Content Determination}

The content of total lipids in the filtrates of the culture media was investigated. Lipids were extracted by continuous liquid/liquid extraction with refluxing diethyl ether using the following procedure. Diethyl ether was added in a weighed extraction cup and the temperature control was set at $80^{\circ} \mathrm{C}$. The extraction process included three steps: boiling, rinsing, and solvent evaporation from the cup. This procedure was applied three times per sample to ensure total recovery of lipids. Each extract was equilibrated at room temperature for $30 \mathrm{~min}$. The mass of the extracted lipids was measured on an analytical balance.

\section{Results and Discussion}

The development of a nutrient medium for growing any producer consists of two stages: the choice of nutrition sources and the establishment of their optimal quantitative ratio. Already at the stage of screening nutrition source, it is desirable to obtain a sufficiently high yield of the target product. This is a prerequisite for achieving success at the stage of optimizing the quantitative ratio of nutrient medium ingredients. When choosing sources of carbon and nitrogen, it is advisable to compare their paired combinations, and not to study each nutrition source separately. This approach makes it possible to identify combinations with positive interactions of nutrient media ingredients.

\subsection{Effect of Carbon and Nitrogen Sources on Accumulation of Flammulina velutipes Submerged Biomass}

The growth of fungal mycelium in submerged cultures is largely affected by carbon and nitrogen sources. The development of the composition of a liquid nutrient medium for submerged cultivation of $F$. velutipes in order to obtain its biomass began with the choice of carbon and nitrogen sources. The yield of fungal biomass was evaluated on media containing glucose, soybean oil or molasses as a carbon source and soybean meal, peptone, corn extract or yeast extract as a nitrogen source. The culture media containing all possible paired combinations of these power sources were studied. The concentrations of carbon and nitrogen sources are shown in Table 1 . The studied media also included $2.5 \mathrm{wt}$. \% $\mathrm{KH}_{2} \mathrm{PO}_{4}$ and 0.25 wt. $\% \mathrm{MgSO}_{4}$. The duration of the $F$. velutipes biomass accumulation process was determined earlier and amounted to 6 days.

Table 1. Concentrations of carbon and nitrogen sources $(\mathrm{g} / \mathrm{L})$ in liquid media for submerged cultivation of $F$. velutipes.

\begin{tabular}{ccccccc}
\hline \multicolumn{3}{c}{ Carbon Sources } & \multicolumn{4}{c}{ Nitrogen Sources } \\
\hline Glucose & $\begin{array}{c}\text { Soybean } \\
\text { Oil }\end{array}$ & Molasses & $\begin{array}{c}\text { Soybean } \\
\text { Meal }\end{array}$ & Peptone & $\begin{array}{c}\text { Corn } \\
\text { Extract }\end{array}$ & $\begin{array}{c}\text { Yeast } \\
\text { Extract }\end{array}$ \\
\hline 20 & 20 & 32 & 10 & 10 & 12 & 5 \\
\hline
\end{tabular}

The results obtained (Figure 1) showed that the yield of the submerged biomass of F. velutipes varied from $7.0 \pm 0$ to $22.7 \pm 0.1 \mathrm{~g} / \mathrm{L}$. To a greater extent, the biomass yield depended on the carbon source. Soybean oil was the most efficient carbon source and soybean meal was the most efficient nitrogen source. Low biomass yield was observed on media containing glucose and peptone, corn extract, or yeast extract. The effectiveness of soybean oil as a carbon source was significantly higher than that of glucose. In our opinion, 
this is due to the higher energy content of soybean oil compared to glucose. For further work, a medium with soybean oil and soybean meal was chosen.

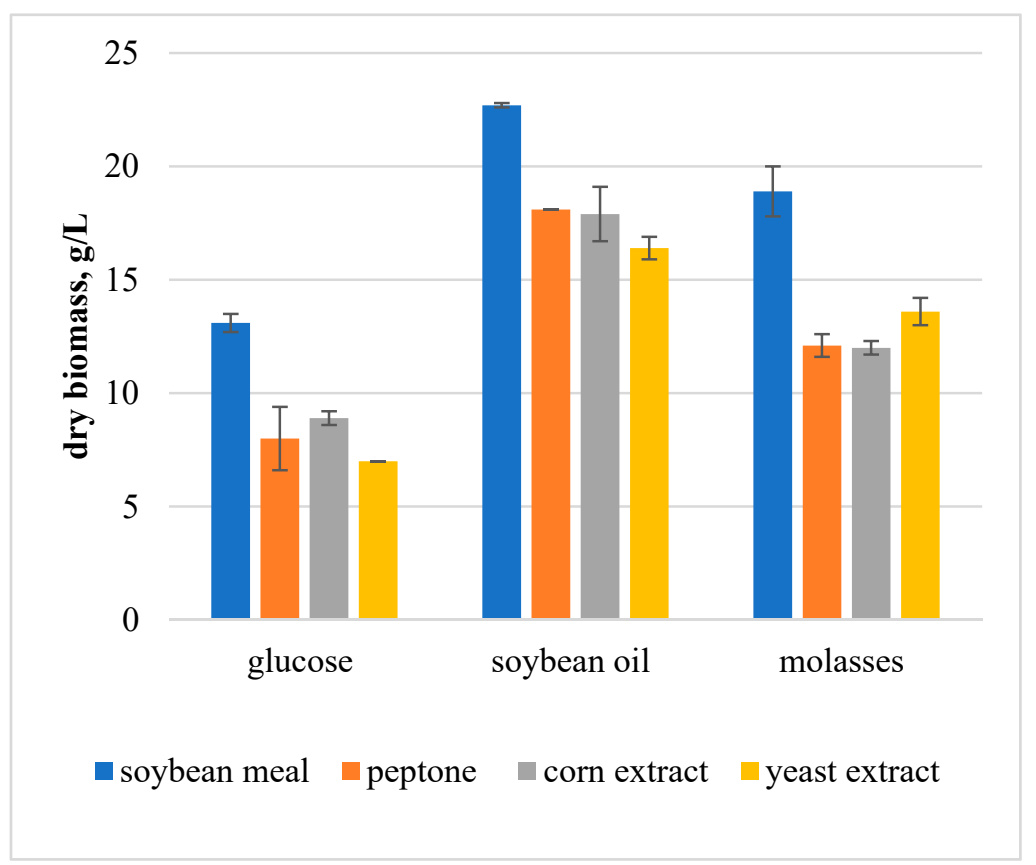

Figure 1. F. velutipes biomass yield on media with different sources of carbon and nitrogen.

The results obtained confirmed that the study of the trophic needs of the fungus culture is a necessary stage in the development of an effective nutrient medium. By examining combinations of carbon and nitrogen sources, we identified both low-efficiency and highefficiency nutrient media. Most nutrient media with glucose, which is a commonly used carbon source, were low-efficiency.

\subsection{Optimization of the Culture Medium for Submerged Cultivation of Flammulina velutipes. Complete Factorial Experiment}

To achieve the maximum biomass yield of F. velutipes, the composition of the nutrient medium with soybean oil and soybean meal was optimized. The full factorial experiment method and the Box-Wilson steep ascent method were used [9]. In the mathematical methods used to design the experiment, the biomass yield of F. velutipes was the response function. In the full factorial experiment, the independent variables were all ingredients of the nutrient medium. The matrix of the full factorial experiment $2^{4}$ included 16 variants of nutrient media, which were all possible combinations of four ingredients on two levels. Additionally, a nutrient medium in which the ingredients were at the 0-level was included in the matrix of the full factorial experiment. The factors indicated were: soybean oil-X1, soybean meal-X2, $\mathrm{KH}_{2} \mathrm{PO}_{4}-\mathrm{X} 3$ and $\mathrm{MgSO}_{4}-\mathrm{X} 4$. Factor levels are shown in Table 2.

Table 2. Levels of factors $(\mathrm{g} / \mathrm{L})$ in a full factorial experiment $2^{4}$.

\begin{tabular}{ccccc}
\hline \multirow{2}{*}{ Factors Level } & \multicolumn{3}{c}{ Factors } \\
\cline { 2 - 5 } & $\mathbf{X 1}$ & $\mathbf{X 2}$ & $\mathbf{X 3}$ & $\mathbf{X 4}$ \\
\hline 1 & 2 & 2 & 0.5 & 0.05 \\
0 & 10 & 10 & 2.5 & 0.25 \\
-1 & 18 & 18 & 4.5 & 0.45 \\
\hline
\end{tabular}

The planning matrix and the results of the full factorial experiment $2^{4}$ are shown in Table 3. The highest yield of $F$. velutipes biomass was obtained on media 4, 8, 12 and 16, 
which include the upper level of soybean oil and soybean meal. The yield of dry F. velutipes biomass on these media was higher than in the best variant of the previous experiment.

Table 3. The planning matrix and the results of the full factorial experiment $2^{4}$.

\begin{tabular}{|c|c|c|c|c|c|c|c|c|c|}
\hline \multirow{3}{*}{$\begin{array}{c}\text { No } \\
1\end{array}$} & \multicolumn{4}{|c|}{ Factors Levels } & \multirow{2}{*}{\multicolumn{3}{|c|}{ Dry Biomass, g/L }} & \multirow{3}{*}{$\begin{array}{c}\text { Mean } \\
\text { Dry Biomass, g/L } \\
4.5\end{array}$} & \multirow{3}{*}{$\frac{\text { MSE * }}{0.3}$} \\
\hline & \multirow{2}{*}{$\begin{array}{r}\mathbf{X 1} \\
-1\end{array}$} & \multirow{2}{*}{$\begin{array}{r}\mathrm{X} 2 \\
-1\end{array}$} & \multirow{2}{*}{$\begin{array}{r}\text { X3 } \\
-1\end{array}$} & \multirow{2}{*}{$\begin{array}{r}\mathbf{X} 4 \\
-1\end{array}$} & & & & & \\
\hline & & & & & 4.1 & 4.8 & 4.7 & & \\
\hline 2 & 1 & -1 & -1 & -1 & 12.7 & 11.4 & 11.2 & 11.8 & 1.3 \\
\hline 3 & -1 & 1 & -1 & -1 & 11.1 & 11.5 & 11.7 & 11.4 & 0.2 \\
\hline 4 & 1 & 1 & -1 & -1 & 25.2 & 26.1 & 26.3 & 25.9 & 0.7 \\
\hline 5 & -1 & -1 & 1 & -1 & 5.2 & 4.3 & 4.5 & 4.7 & 0.4 \\
\hline 6 & 1 & -1 & 1 & -1 & 12.8 & 12.8 & 13.7 & 13.1 & 0.5 \\
\hline 7 & -1 & 1 & 1 & -1 & 12.5 & 12.5 & 11.9 & 12.3 & 0.2 \\
\hline 8 & 1 & 1 & 1 & -1 & 27 & 26.1 & 27.1 & 26.7 & 0.6 \\
\hline 9 & -1 & -1 & -1 & 1 & 4 & 4.6 & 4.5 & 4.4 & 0.2 \\
\hline 10 & 1 & -1 & -1 & 1 & 11.7 & 12.5 & 12.2 & 12.1 & 0.3 \\
\hline 11 & -1 & 1 & -1 & 1 & 12.5 & 12.6 & 12.6 & 12.6 & 0.0 \\
\hline 12 & 1 & 1 & -1 & 1 & 26.6 & 25.9 & 25.6 & 26.0 & 0.5 \\
\hline 13 & -1 & -1 & 1 & 1 & 4.3 & 4.8 & 4.5 & 4.5 & 0.1 \\
\hline 14 & 1 & -1 & 1 & 1 & 12.8 & 12.8 & 13.4 & 13.0 & 0.2 \\
\hline 15 & -1 & 1 & 1 & 1 & 13.5 & 12.3 & 12.9 & 12.9 & 0.7 \\
\hline 16 & 1 & 1 & 1 & 1 & 25.9 & 26.3 & 27.2 & 26.5 & 0.9 \\
\hline 17 & 0 & 0 & 0 & 0 & 15.3 & 15.2 & & 15.3 & 0.005 \\
\hline
\end{tabular}

The data obtained in full factorial experiment $2^{4}$ made it possible to derive the regression coefficients of the factors and their paired interactions. The regression coefficients are shown in Table 4 . The resulting regression equation, taking into account the factors and their paired interactions, was as follows (Equation (1)):

$$
\begin{gathered}
\mathrm{Y}=13.97941+5.48750 \mathrm{X} 1+5.38750 \mathrm{X} 2+0.31250 \mathrm{X} 3+0.10000 \mathrm{X} 4+1.50000 \mathrm{X} 1 \cdot \mathrm{X} 2+0.12500 \mathrm{X} 1 \cdot \mathrm{X} 3- \\
0.07917 \mathrm{X} 1 \cdot \mathrm{X} 4+0.10417 \mathrm{X} 2 \cdot \mathrm{X} 4-0.08750 \mathrm{X} 3 \cdot \mathrm{X} 4
\end{gathered}
$$

Table 4. Regression coefficients and $t$-test values.

\begin{tabular}{ccccc}
\hline \multirow{2}{*}{ Mean/Interc. } & Regress. Coeff. & Std. Err. & $\boldsymbol{t} \mathbf{( 6 )}$ & $\boldsymbol{p}$ \\
\cline { 2 - 5 } & $\mathbf{1 3 . 9 7 9 4 1}$ & $\mathbf{0 . 1 5 1 0 7 7}$ & $\mathbf{9 2 . 5 3 1 7 6}$ & $\mathbf{0 . 0 0 0 0 0 0}$ \\
\hline X1 & $\mathbf{5 . 4 8 7 5 0 *}$ & 0.311453 & 35.23806 & 0.000000 \\
X2 & $\mathbf{5 . 3 8 7 5 0 *}$ & 0.311453 & 34.59591 & 0.000000 \\
X3 & 0.31250 & 0.311453 & 2.00672 & 0.091569 \\
X4 & 0.10000 & 0.311453 & 0.64215 & 0.544514 \\
X1 by X2 & $\mathbf{1 . 5 0 0 0 0 *}$ & 0.311453 & 9.63227 & 0.000072 \\
X1 by X3 & 0.12500 & 0.311453 & 0.80269 & 0.452768 \\
X1 by X4 & -0.07917 & 0.311453 & -0.50837 & 0.629339 \\
X2 by X3 & 0.00000 & 0.311453 & 0.00000 & 1.000000 \\
X2 by X4 & 0.10417 & 0.311453 & 0.66891 & 0.528436 \\
X3 by X4 & -0.08750 & 0.311453 & -0.56188 & 0.594547 \\
\hline
\end{tabular}

* Regression coefficients of statistically significant factors and factor interaction.

The regression equation does not contain the $\mathrm{X} 2 \mathrm{X} 3$ term because its regression coefficient is 0 .

The statistical significance of the regression coefficients was determined by the value of the $t$-test (Student's test). Figure 2 displays a Pareto diagram showing the quantitative ratios of the $t$-test values of the studied factors in descending order at a significance level of 0.05 . 


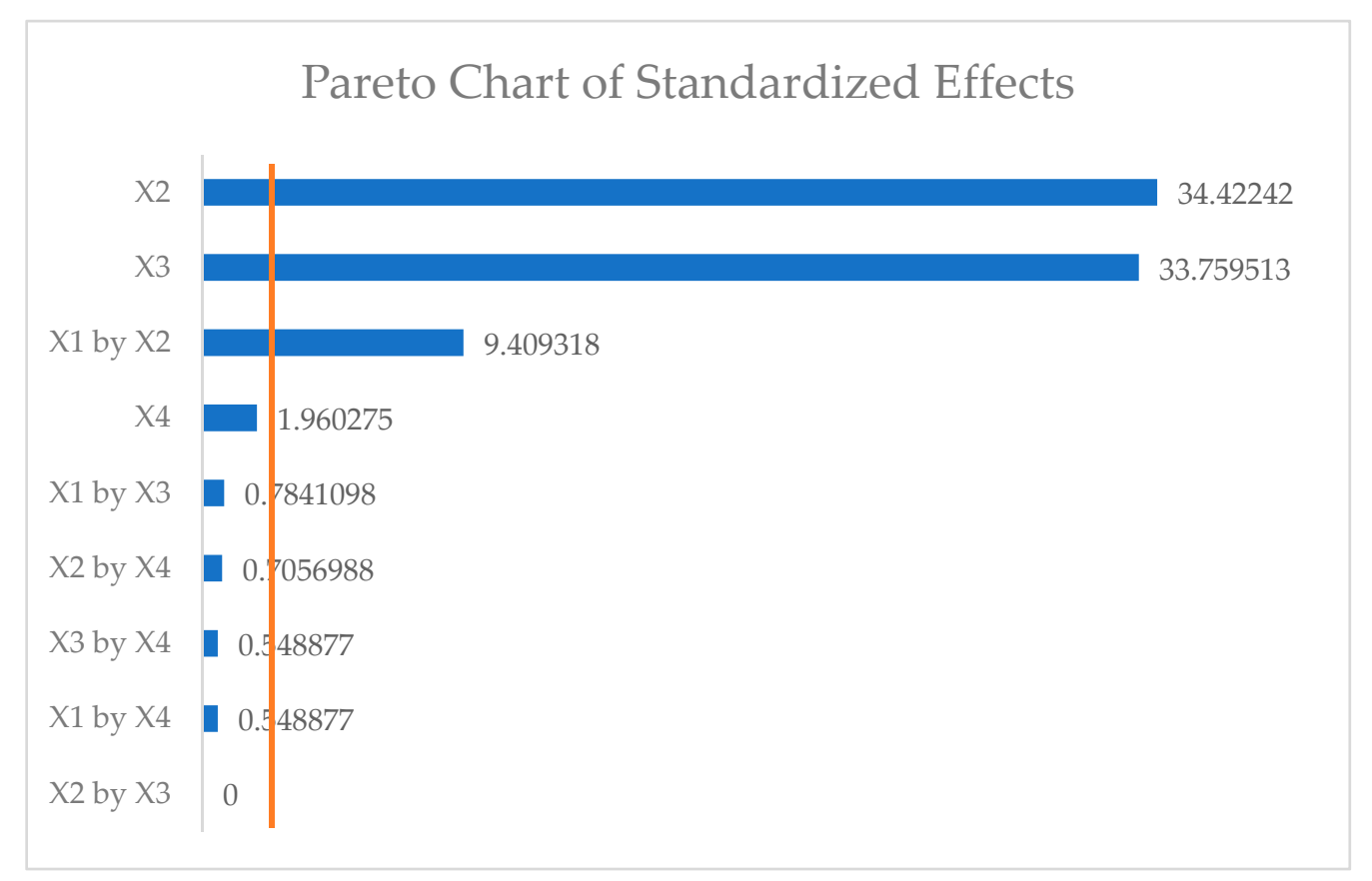

Figure 2. Pareto chart, the $t$-test values of factors and their paired interactions $(p=0.5)$.

The results presented in Table 4 and Figure 2 showed that soybean oil and soybean meal had a statistically significant effect on the biomass yield of F. velutipes. The investigated concentrations of these nutrition sources were in the limiting region. Therefore, to increase the yield of $F$. velutipes biomass, it was necessary to increase their content in the nutrient medium. Since the regression coefficients of factors X1 and X2 were very close in value, it was reasonable to increase the concentration of soybean oil and soybean meal in the sleep ascent experiment with the same step. The results obtained also showed the presence of positive interaction between soybean oil and soybean meal. The limiting factors involved in paired positive interaction reinforce each other's effect. Consequently, their positive interaction reveals the need to increase their concentrations in the nutrient medium. In relation to our results, this positive interaction did not change the strategy for conducting subsequent experiments.

The response surface reflecting the yield of fungal biomass was plotted according to the results of a full factorial experiment $2^{4}$. To construct the response surface, two statistically significant factors were used: soybean oil and soybean meal (Figure 3). Figure 3 shows that in the full factorial experiment, the maximum biomass yield of $F$. velutipes was not achieved. To complete the movement to the extremum, an experiment using the steep ascent method was required. This method consists of the simultaneous change in the values of all statistically significant factors at each step of the experiment. 


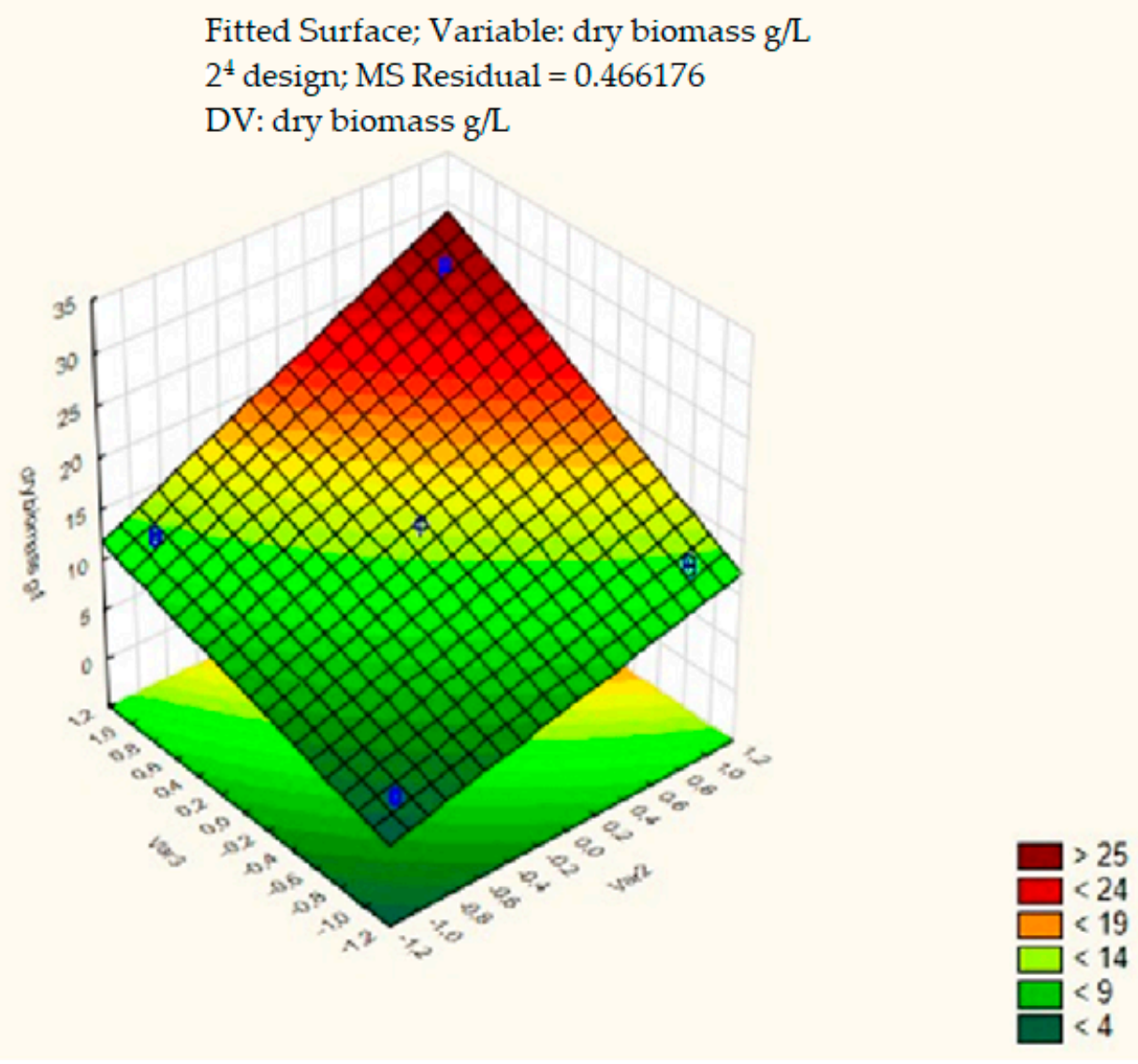

Figure 3. Response surface. The yield of F. velutipes biomass depending on the concentrations of soybean oil (Var 2) and soybean meal (Var 3).

3.3. Optimization of the Nutrient Medium for Deep Cultivation of Flammulina velutipes Steep Ascent Experiments

Two experiments were carried out using the steep ascent method. At each step of the experiments the concentrations of two statistically significant factors, soybean oil and soybean meal were sequentially increased. Since their regression coefficients were close in value, the step of variation of these factors was the same. In the first experiment it was $2 \mathrm{~g} / \mathrm{L}$, in the second $-1 \mathrm{~g} / \mathrm{L}$. The content of mineral salts in the nutrient media was constant: $\mathrm{KH}_{2} \mathrm{PO}_{4} 2 \mathrm{~g} / \mathrm{L}, \mathrm{MgSO}_{4} 0.25 \mathrm{~g} / \mathrm{L}$. The results are shown in Figure 4 . The first experiment included nine nutrient media. The first step culture medium contained ingredients at 0-level. The first experiment showed that an increase in the content of soybean oil and soybean meal in the nutrient medium leads to magnification in the biomass yield of F. velutipes. The revealed relationship between the studied concentration of factors and the biomass yield was linear. There was no evidence of reaching the maximum biomass yield in the first experiment.

It is known that the optimization of nutrient media not only leads to an increase in the yield of the target product but can shorten the duration of the cultivation process. To test this assumption, we conducted a comparative study of the accumulation of $F$. velutipes biomass on the initial nutrient medium (No. 1) and on the best nutrient medium (No. 9) of the first experiment using the steep ascent method. According to the results obtained (Figure 5), the maximum biomass yield of the fungus was noted on the 5th day of the cultivation process. Thus, the duration of the submerged cultivation of F. velutipes was reduced by 1 day. In further experiments, F. velutipes were cultured for 5 days. 


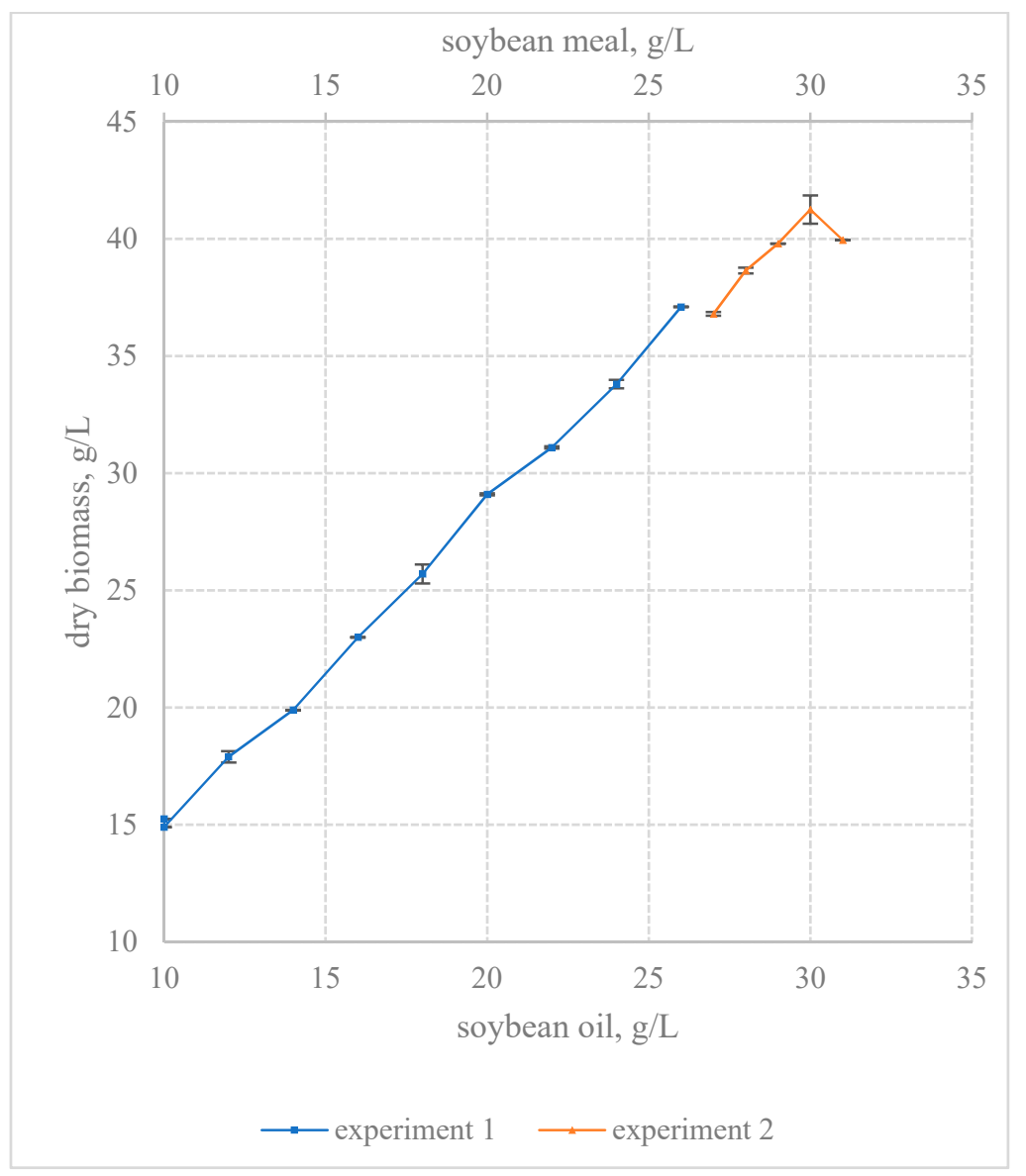

Figure 4. Steep ascent experiments. The yield of F. velutipes biomass with increasing concentrations of soybean oil and soybean meal in the nutrient medium.

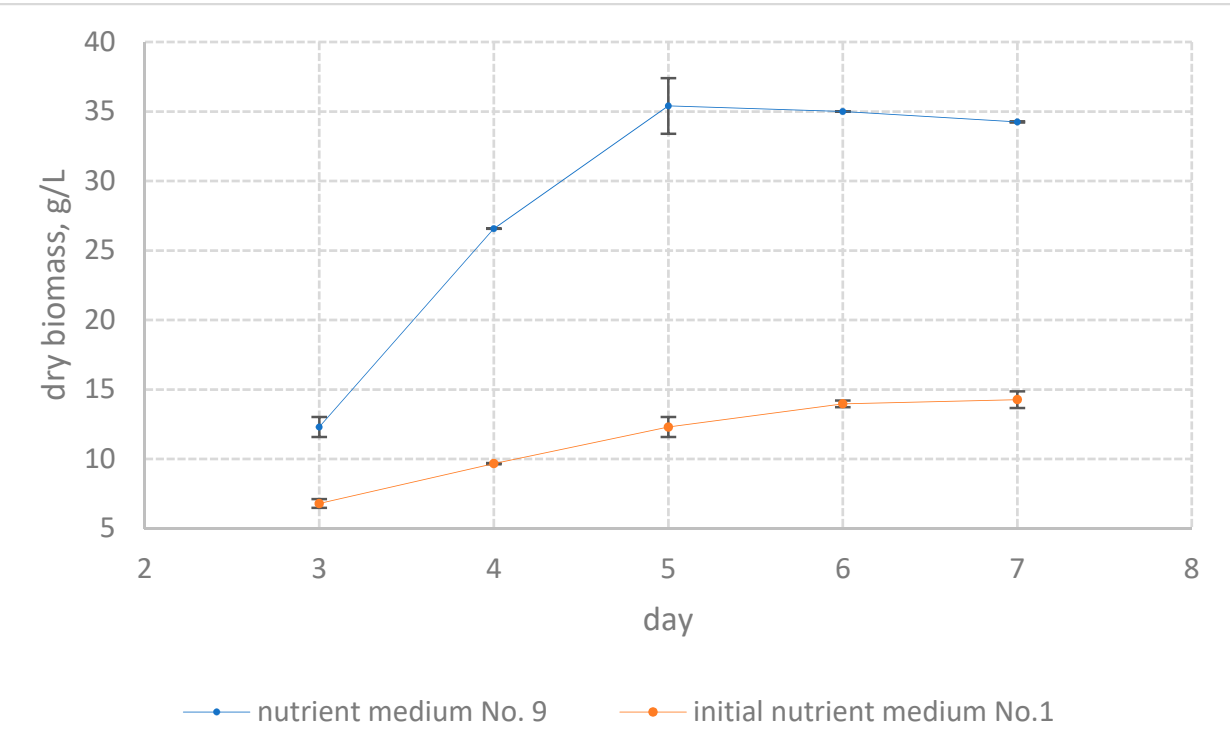

Figure 5. Accumulation of $F$. velutipes biomass during submerged cultivation on the initial nutrient medium No. 1 and nutrient medium No. 9 of the first steep ascent experiment.

Therefore, a second experiment was carried out using the steep ascent method. It continued to increase the content of soybean oil and soybean meal. However, the step of variation in the second experiment was halved. The second experiment included five 
culture media (Figure 5). The last nutrient medium of the first experiment was chosen as the first nutrient medium of the second ones. The maximum yield of the submerged biomass of F. velutipes was achieved at step 4 . It reached $41.25 \pm 0.61 \mathrm{~g} / \mathrm{L}$. The submerged culture obtained at step 4 of the steep ascent was further studied. Light and electron microscopy did not reveal any residues of soybean meal in the fungus biomass or in the filtrate of the culture medium. The filtrate of the culture medium did not contain lipids, which indicated the complete utilization of soybean oil. A further increase in the content of soybean oil and soybean meal in the nutrient medium led to a decrease in the accumulation of the fungus biomass. Thus, it was proved that the maximum yield of the fungus biomass is achieved by submerged cultivation on a nutrient medium containing $30 \mathrm{~g} / \mathrm{L}$ of soybean oil and $30 \mathrm{~g} / \mathrm{L}$ of soybean meal. The use of the mathematical design of the experimental approach made it possible to increase the yield of the submerged biomass of F. velutipes by $82 \%$ in comparison with the initial medium.

\subsection{Micromorphology of Flammulina velutipes Submerged Mycelium}

Efficient production of fungal biomass or metabolite usually correlates with certain micromorphological characteristics of the producer's mycelium. In our study scanning electron microscopy was used to examine a 5-day-old submerged mycelium of $F$. velutipes grown on optimized media (Figure 6). The fungus grew in submerged culture in the form of small spherical pellets with a diameter of 1-2 mm. The pellets had outgrowths of hyphae bundles. The surface of the pellets was loose; the inner part was filled with hyphae tightly adjacent to one another. The center of the pellets had no cavity. In this way, F. velutipes pellets differed from pellets formed by many other species of basidiomycetes. As an example, a cut of a Grifola frondosa pellet with cavities inside the pellet is shown. F. velutipes pellets were formed by septate hyphae with clamp connections. The clamp connections are a characteristic feature of the basidiomycetes hyphae.

The study of the morphological characteristics of the submerged mycelium of F. velutipes helped to explain the achieved high yield of fungal biomass on an optimized nutrient medium. The loose surface and small size of pellets created conditions for active diffusion of nutrients and oxygen, which ensured the activation of growth and biosynthesis processes. The small size of the pellets ensured the maximum filling of the entire volume of the culture medium. The absence of cavities inside the pellets increased their density and led to an increase in the mass of the grown mycelium.

There is reason to believe that the small size of the pellets is associated with the presence of soybean meal in the nutrient medium. Currently, one of the leading modern methods of morphological engineering is microparticle-enhanced cultivation (MPEC). This method, developed for filamentous fungi, consists in adding microparticles of inorganic compounds (magnesium silicate, aluminum oxide, titanium oxide) to nutrient media, which leads to a decrease in the size of pellets and, as a consequence, to an increase in the yield of secondary metabolites, enzymes or biomass [10]. The use of soybean meal can be attributed to the MPEC method since soybean meal is composed of microparticles. The difference between inorganic and organic microparticles is that the inorganic microparticles remain unchanged until the end of the cultivation process, while the organic microparticles are gradually utilized by the filamentous fungus during the cultivation process. Therefore, it can be concluded that organic microparticles of soybean meal influenced the initial phase of the formation of F. velutipes pellets. There is evidence that inorganic microparticles also affect the mycelium of fungi in the early stages of their cultivation. A study by Driouch H. et al. [11] showed that talc and aluminum oxide microparticles change the morphology of Aspergillus niger submerged in the very early stage of the cultivation process. Kowalska A. et al. [12] studied the species of filamentous fungi that differ in the mechanisms of pellet formation. It was shown that the presence or absence of the effect of adding aluminum oxide depended on the mechanism of the formation of the pellets. This means that the influence of these inorganic microparticles is carried out in the early stages of filamentous fungi cultivation. 


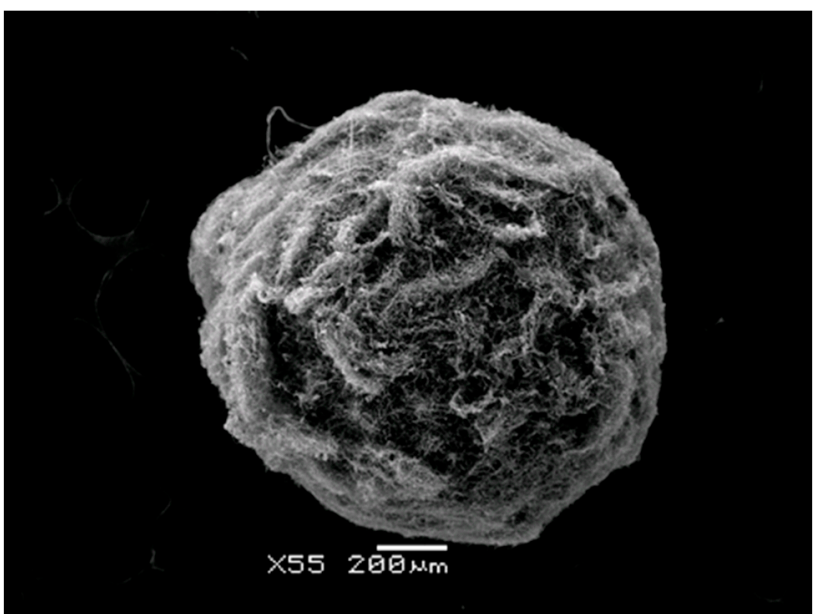

(a)

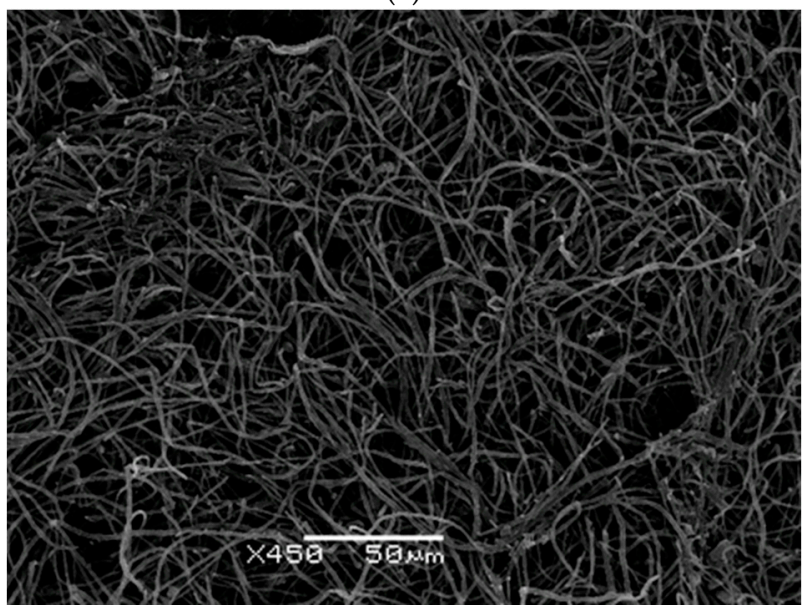

(c)

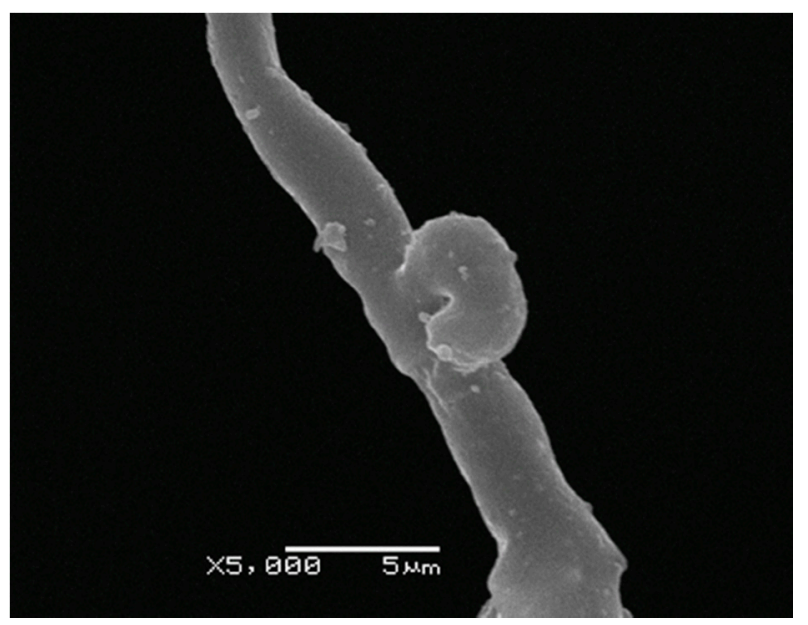

(e)

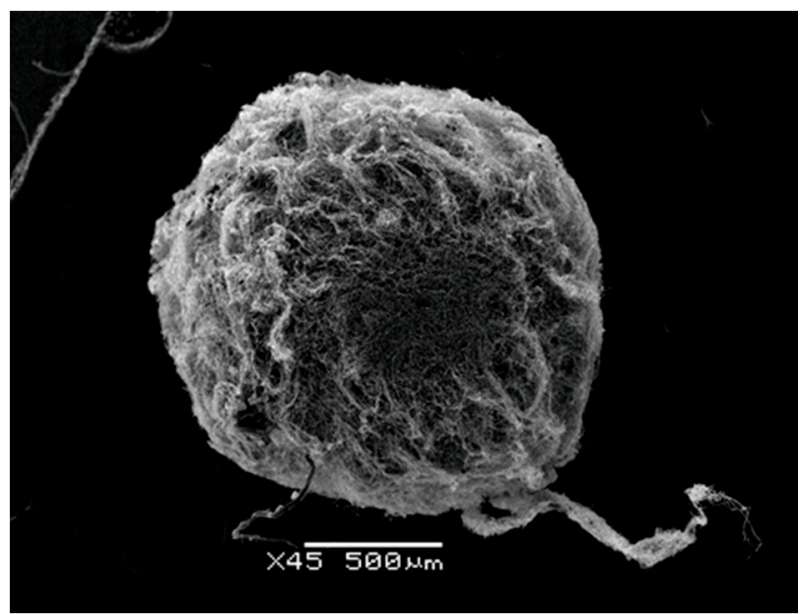

(b)

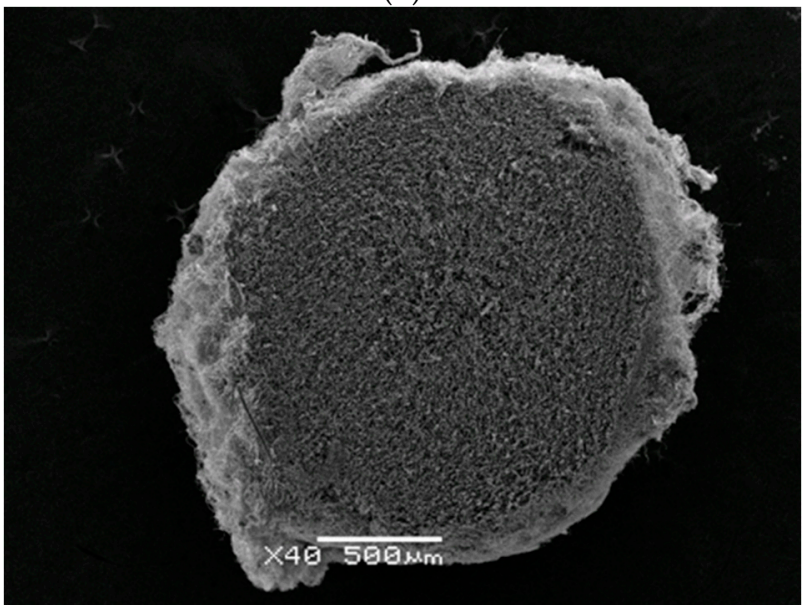

(d)

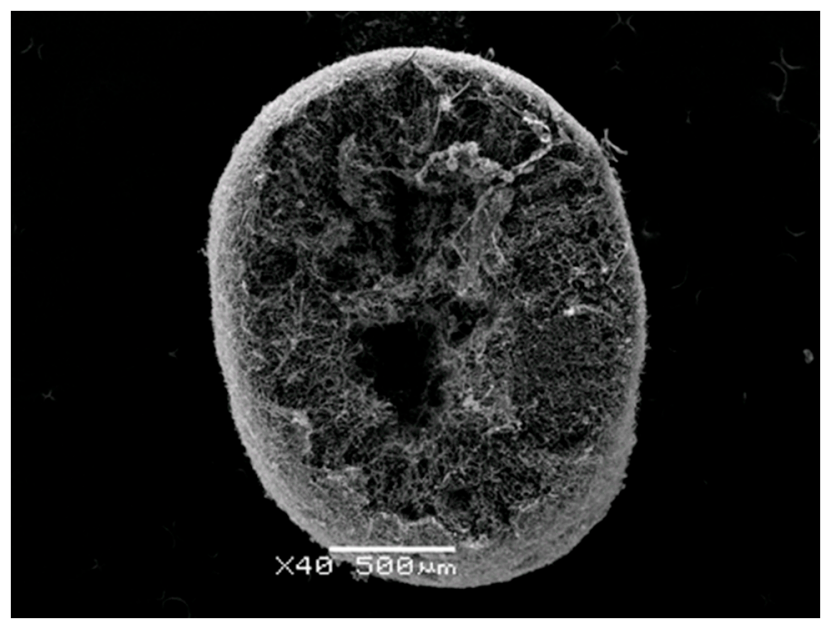

(f)

Figure 6. Scanning electron microscopy of F. velutipes submerged mycelium: $(\mathbf{a}, \mathbf{b})$ - general view of the pellets, (c) - pellet surface, (d)—pellet slice, (e)—hyphae with clamp connection, (f) —slice of G. frondosa pellet.

\section{Conclusions}

The development of a nutrient medium for submerged cultivation of the selected F. velutipes strain was carried out in two stages. The purpose of the first phase was to select carbon and nitrogen sources. At the second stage, the composition of the nutrient medium was optimized using the methods of mathematical planning of the experiment. 
When choosing the sources of carbon and nitrogen, their paired effect on the yield of the fungus biomass was assessed. Although widely used nutrient sources were tested in the experiment, the biomass yield could differ by three-time. It should be emphasized that culture media containing such a commonly used carbon source as glucose were of low efficiency. The results obtained show that the stage of selection of nutrient sources is necessary for the development of an efficient nutrient medium. Comparing paired combinations of carbon and nitrogen sources, as opposed to examining each nutrient source separately, allows for a quick and complete assessment of the nutrient needs of the producer.

When carrying out the optimization of the culture medium, the $2^{4}$ full factorial design and the Box-Wilson steep ascent method were used. The presence of only four ingredients in the nutrient medium made it possible to carry out a full factorial experiment since the experimental matrix contained only 16 nutrient media options. Based on the results of a full factorial experiment, statistically significant factors were identified. Soybean oil and soybean meal were those limiting factors. There was also a positive interaction between soybean oil and soybean meal. The results obtained substantiated the need to increase the concentrations of significant factors. A simultaneous increase in the content of soybean oil and soybean meal in the nutrient medium was carried out using the steep ascent method. The optimal concentrations of these factors were determined to ensure the maximum biomass yield of F. velutipes. Optimization of the nutrient medium composition provided not only an increase in the yield of the submerged biomass of F. velutipes up to $41 \mathrm{~g} / \mathrm{L}$, but also a reduction in the duration of the cultivation process by one day (to 5 days). A similar reduction in the duration of the process was noted by us earlier when optimizing the nutrient medium for the cultivation of the basidiomycete Ganoderma lucidum [13].

Comparison of our results with other published data is difficult due to differences in the conditions of submerged cultivation of F. velutipes. According to Florez et al. [14], the yield of submerged biomass of F. velutipes does not exceed $9 \mathrm{~g} / \mathrm{L}$. However, this low yield is most likely associated not only with the composition of the nutrient medium but also with insufficient aeration of the culture, since submerged cultivation was carried out on a rotary shaker at $100 \mathrm{rpm}$. Hassan et al. (2010) obtained the maximum yield of the submerged biomass of F. velutipes (up to $11.86 \mathrm{~g} / \mathrm{L}$ ) using a nutrient medium with glucose and yeast extract. [15]. The authors consider these sources of carbon and nitrogen to be the most favorable for submerged cultivation of F. velutipes. This conclusion contradicts our results, according to which the nutrient medium with glucose and yeast extract is the least suitable for obtaining the biomass of the fungus. However, a set of carbon and nitrogen sources with the exception of glucose, yeast extract and peptone in Hassan et al. differs from the set we tested. The duration of F. velutipes submerged cultivation in the cited works is 11 [14] and 15 days [14], which significantly exceeds the duration of cultivation of the fungus on the medium developed by us.

The studied strain of F. velutipes is undoubtedly one of the most biomass-productive cultures. Similar values were obtained in our studies only with the Hypsizygus ulmarius strain, the yield of submerged biomass of which reached 32-33 g/L [16]. The study of the micromorphology of the submerged mycelium of F. velutipes allows us to understand the reasons for the high productivity of the used strain. Under optimized conditions, the studied strain formed small pellets with a loose surface and a completely filled center. Such properties of pellets provide a high level of mass transfer and contribute to the maximum possible filling of the culture medium volume with the biomass of the fungus. Soybean meal seems to play a significant role in the formation of pellets of small size and established structure. In our opinion, its application can be considered as a special case of one of the morphological engineering methods, namely, microparticle-enhanced cultivation.

Author Contributions: Conceptualization, methodology, L.K.; software, A.G.; formal analysis, L.K., A.G.; investigation, L.K., M.S., A.G., N.A., M.Y.; writing-original draft preparation, L.K., N.A.; writing-review and editing, L.K.; project administration, L.K. All authors have read and agreed to the published version of the manuscript. 
Funding: This research received no external funding.

Institutional Review Board Statement: Not applicable.

Informed Consent Statement: Informed consent was obtained from all subjects involved in the study.

Conflicts of Interest: The authors declare no conflict of interest.

\section{References}

1. Wang, Y.; Zhang, H. Advances in the extraction, purification, structural-property relationships and bioactive molecular mechanism of Flammulina velutipes polysaccharides: A review. Int. J. Biol. Macromol. 2020, 167, 528-538. [CrossRef] [PubMed]

2. Zhang, Y.; Li, H.; Yang, X.; Jin, G. Cognitive-enhancing effect of polysaccharides from Flammulina velutipes on Alzheimer's disease by compatibilizing with ginsenosides. Int. J. Biol. Macromol. 2018, 112, 788-795. [CrossRef] [PubMed]

3. Zhang, T.; Ye, J.; Xue, C.; Wang, Y.; Liao, W.; Mao, L.; Yuan, M.; Lian, S. Structural characteristics and bioactive properties of a novel polysaccharide from Flammulina velutipes. Carbohydr. Polym. 2018, 197, 147-156. [CrossRef] [PubMed]

4. Zhao, C.; Zhao, K.; Liu, X.; Huang, Y.-F.; Liu, B. In Vitro Antioxidant and Antitumor Activities of Polysaccharides Extracted from the Mycelia of Liquid-Cultured Flammulina velutipes. Food Sci. Technol. Res. 2013, 19, 661-667. [CrossRef]

5. Liu, Y.; Bastiaan-Net, S.; Wichers, H.J. Current Understanding of the Structure and Function of Fungal Immunomodulatory Proteins. Front. Nutr. 2020, 7, 132. [CrossRef] [PubMed]

6. Fukushima-Sakuno, E. Bioactive small secondary metabolites from the mushrooms Lentinula edodes and Flammulina velutipes. J. Antibiot. 2020, 73, 687-696. [CrossRef] [PubMed]

7. Krasnopolskaya, L.; Shuktueva, M.; Avtonomova, A.; Yarina, M.; Dzhavakhyan, B.; Isakova, E.; Bukhman, V. Antitumor and Antioxidant Properties of Water-Soluble Polysaccharides from Submerged Mycelium of Flammulina velutipes. Antibiot. I Khimioter 2016, 61, 16-20.

8. Almyasheva, N.R.; Yarina, M.S.; Golyshkin, A.V.; Dzhavakhyan, B.R.; Krasnopolskaya, L.M. Antioxidant Properties of WaterSoluble Polysaccharides and Ethanolic Extracts of Xylotrophic Basidiomycetes Mycelium. Antibiot. I Khimioter 2017, 62, 8-12.

9. Maksimov, V.N. Multifactorial Experiments in Biology. Mosk. Gos. Univ.: Moscow, Russia, 1980; pp. 280p.

10. Antecka, A.; Bizukojc, M.; Ledakowicz, S. Modern morphological engineering techniques for improving productivity of filamentous fungi in submerged cultures. World J. Microbiol. Biotechnol. 2016, 32, 1-9. [CrossRef] [PubMed]

11. Driouch, H.; Sommer, B.; Wittmann, C. Morphology engineering of Aspergillus niger for improved enzyme production. Biotechnol. Bioeng. 2010, 105, 1058-1068. [CrossRef] [PubMed]

12. Kowalska, A.; Boruta, T.; Bizukojc, M. Morphological evolution of various fungal species in the presence and absence of aluminum oxide microparticles: Comparative and quantitative insights into microparticle-enhanced cultivation (MPEC). MicrobiologyOpen 2018, 7, e00603. [CrossRef] [PubMed]

13. Avtonomova, A.V.; Krasnopol'Skaya, L.M.; Maksimov, V.N. Optimization of nutrient medium for submerged cultivation of Ganoderma lucidum (Curt.: Fr.) P. Karst. Microbiology 2006, 75, 148-153. [CrossRef]

14. Flórez, D.F.R.; Chegwin-Angarita, C.; Melo, O.O.; Nieto-Ramírez, I.J. Estudio comparativo de la producción de biomasa en fermentación superficial y en estado líquido de macromicetos con diferentes fuentes nutrimentales (Comparative study of the production of biomass in surface fermentation and in the liquid state of macromycetes with different nutrient sources). Rev. Colomb. Biotecnol. 2019, 21, 39-46. [CrossRef]

15. Hassan, F.R.H.; Ghada, M.M.; El-Kady, A.T.M. Mycelial biomass production of Enoke Mushroom (Flammulina velutipes) by submerged culture. Aust. J. Basic Appl. Sci. 2012, 6, 603-610.

16. Krasnopolskaya, L.; Leontieva, M.; Avtonomova, A.; Isakova, E.; Belitskii, I.; Usov, A.; Buchman, V. Antitumor properties of submerged cultivated biomass and extracts of medicinal mushrooms of genus Hypsizygus Singer. Int. J. Med. Mushrooms 2008, 10, 25-35. [CrossRef] 